\title{
Advanced Repetitive Controller to Improve the Voltage Characteristics of Distributed Generation with Nonlinear Loads
}

\author{
Quoc-Nam Trinh ${ }^{*}$ and Hong-Hee Lee ${ }^{\dagger}$ \\ $\dagger^{*}$ School of Electrical Engineering, University of Ulsan, Ulsan, Korea
}

\begin{abstract}
This paper presents an enhanced control strategy which consists of a proportional-integral controller and a repetitive controller (RC) for improving the voltage performance of distributed generation (DG) under nonlinear load conditions. The proposed voltage controller is able to maintain a sinusoidal voltage at the point of common coupling (PCC) of the DG regardless of the harmonic voltage drop in the system impedance due to nonlinear load currents. In addition, by employing the delay time of the RC at one-sixth of the fundamental period, the proposed RC can overcome the slow response drawback of the traditional PI-RC. The proposed control strategy is analyzed and the design of the RC is presented in detail. The feasibility of the proposed control strategy is verified through simulation and experimental results.
\end{abstract}

Key words: Distributed generation, Harmonic compensation, Nonlinear load, Repetitive controller

\section{INTRODUCTION}

The widespread use of traditional fossil fuels such as oil, coal, and gas results in negative environmental ramifications such as air and water pollution, greenhouse gas emissions, and global climate change. In addition, the huge demand for electricity in developing countries has made these issues even more serious. Renewable energy sources such as wind, solar, and fuel cells have been considered as potential candidates to replace traditional fossil energy sources, and there has been considerable worldwide research on renewable energy during the last few decades. Recently, a large number of renewable energy sources have been integrated into power distribution systems in the form of distributed generation (DG) [1]. Most DG systems have been developed to operate in the grid-connected mode with various operational functions such as power control, harmonic current mitigation, and reactive power compensation [2]-[4]. In addition, the stand-alone (isolated) operation should also be considered in order to exploit the full potential of DG systems. In the stand-alone mode, a DG should deliver a sinusoidal voltage at the point of

Manuscript received Sep. 21, 2012; revised Feb. 5, 2013

Recommended for publication by Associate Editor Jun-Keun Ji.

${ }^{\dagger}$ Corresponding Author: hhlee@mail.ulsan.ac.kr

Tel: +82-52-259-2187, Fax: +82-52-259-1686, University of Ulsan

* School of Electrical Engineering, University of Ulsan, Korea

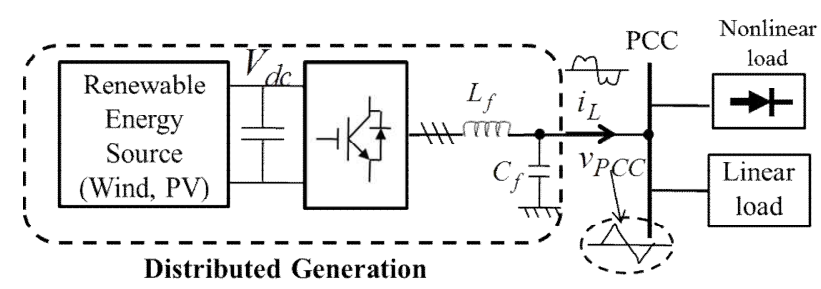

Fig. 1. Configuration of a DG system connected with loads at the PCC.

common coupling (PCC) with a constant magnitude and frequency regardless of the load variation and type of load.

Under balanced linear load conditions, a proportionalintegral (PI) controller in the synchronous reference frame is sufficient to produce a sinusoidal output voltage at the PCC [5]-[7]. However, if unbalanced and/or nonlinear loads are connected at the PCC, the PI controller is no longer able to maintain a sinusoidal PCC voltage. Generally, nonlinear loads draw harmonic currents into the system and cause a distorted voltage drop in the system impedance. Consequently, the PCC voltage of the DG becomes non-sinusoidal as shown in Fig. 1. This non-sinusoidal voltage condition has harmful effects on the other loads connected at the PCC.

In order to supply a pure sinusoidal output voltage at the PCC under a nonlinear load, the inverter must generate a distorted voltage to compensate the harmonic voltage drop in the system impedance. To fulfill that goal, several advanced 
control strategies have been developed [8], [9]. In [8], the authors attempted to improve the PCC voltage performance by using a bank of PI controller where each of the PI controllers is used to regulate the magnitude of a single harmonic voltage. In this control method, many PI controllers and coordinate transformations are required to compensate all of the harmonic voltages, which make the control strategy too complex. Another control method for handling unbalanced and non-linear loads has been developed for DG systems, which combines resonant and predictive controllers [9]. By implementing the control scheme in the stationary reference frame, coordinate transformations are eliminated. However, since each of the resonant controllers is capable of compensating only one specific harmonic voltage, a large number of resonant controllers must be used if all of the harmonic voltages must be compensated. In addition, this control strategy requires the measurement of the load current and a current control loop, resulting in a complex control scheme.

Repetitive controllers (RC) are an effective solution for overcoming the complexity of multiple resonant controllers. The RC, which was originally developed from the internal model principle, is capable of achieving zero errors in tracking periodic signals, e.g., harmonic voltages, by using only a simple delay unit [10]. In addition, since an RC can provide a similar behavior as that of a bank of resonant controllers, a large number of harmonic currents/voltages can be simultaneously compensated by using only one RC [11]. Due to this advantage, RCs have been applied to various applications such as uninterruptable power supplies (UPS) [10]-[13], PFC boost converters [14], [15], and active power filters (APFs) [16]-[18]. Even though RCs have good steady-state performance in harmonic rejection, the traditional PI-RC has a poor dynamic response due to a long delay time, i.e., one period of the fundamental frequency (fundamental period). Particularly, in DG systems where the load conditions change frequently, it is difficult to guarantee that the PCC voltage is always sinusoidal with the traditional PI-RC because of its poor dynamic response. In addition, traditional PI-RC were developed to compensate all harmonic components (odds and evens), but it is unnecessary to compensate even harmonics since most AC systems have only odd harmonics. In particular, in three-phase systems, since only the $(6 n \pm 1)$ th $(n=1,2,3 \ldots)$ harmonic components exist, an RC should be developed to compensate only those specific components to avoid the possibility of amplifying or re-injecting other harmonics into the PCC voltage.

In this paper, in order to overcome the drawbacks of the traditional PI-RC, a new RC is developed for three-phase systems. In the proposed $\mathrm{RC}$, the time delay is limited to one-sixth of the fundamental period so that the proposed RC takes into account only the $(6 n \pm 1)$ th $(n=1,2,3 \ldots)$ harmonic voltage drops caused by nonlinear loads on the system. As a

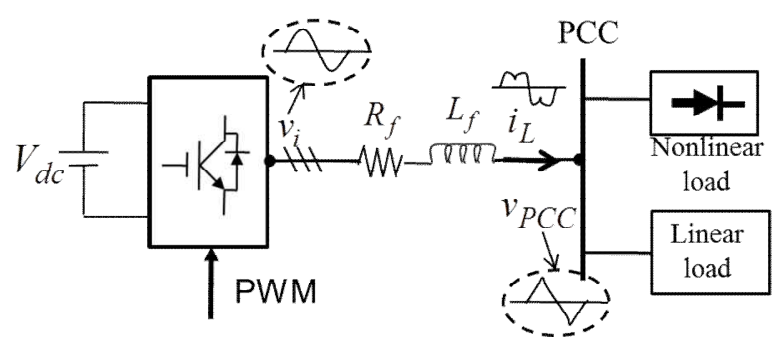

Fig. 2. Connection interface of a DG with various loads.

result, the PCC voltage of the DG is regulated to be a sinusoidal waveform irrespective of the presence of nonlinear loads. In addition, the proposed RC can overcome the slow response of the traditional PI-RC since the time delay of the proposed RC is reduced by six times. The proposed control strategy is analyzed and the design of the RC is presented in detail. The feasibility of the proposed control strategy is verified through simulation and experimental results.

\section{VOLTAGE CONTROL SCHEME}

\section{A. Effect of Nonlinear Loads on PCC Voltage}

The connection interface of a DG with various types of loads, including linear and nonlinear loads, is shown in Fig. 2. In Fig. 2, $R_{f}$ and $L_{f}$ are the resistance and inductance of the line impedance, respectively. Based on Fig. 2, the PCC voltage $\left(v_{P C C}\right)$ of a DG can be calculated as

$$
v_{P C C}=v_{i}-R_{f} i_{L}-L_{f} \frac{d i_{L}}{d t},
$$

where $v_{i}=v_{i 1}$ is the output voltage of the inverter, which is assumed to have only the fundamental component $\left(v_{i 1}\right)$, and $i_{L}$ is the load current.

The nonlinear load under consideration is a three-phase diode rectifier which draws odd harmonic currents into the system with the $(6 n \pm 1)$ th $(n=1,2,3 \ldots)$ orders of the fundamental frequency $\left(\omega_{0}\right)$. Thus, the PCC voltage of the DG is determined by

$$
\begin{aligned}
v_{P C C}=\left(v_{i 1}-R_{f} i_{L 1}\right. & \left.-L_{f} \frac{d i_{L 1}}{d t}\right) \\
& -\left(R_{f} \sum_{h=6 n \pm 1} i_{L h}+L_{f} \sum_{h=6 n \pm 1} \frac{d i_{L h}}{d t}\right),
\end{aligned}
$$

where $i_{L 1}$ and $i_{L h}$ are the fundamental and harmonic components of the load current, respectively.

As can be seen in (2), even though the inverter can generate a pure sinusoidal output voltage, the PCC voltage becomes distorted due to the harmonic voltage drop in the line impedance. Hence, in order to achieve a pure sinusoidal voltage at the PCC, the inverter must generate a distorted voltage to compensate the harmonic voltage drop in the line impedance as follows 


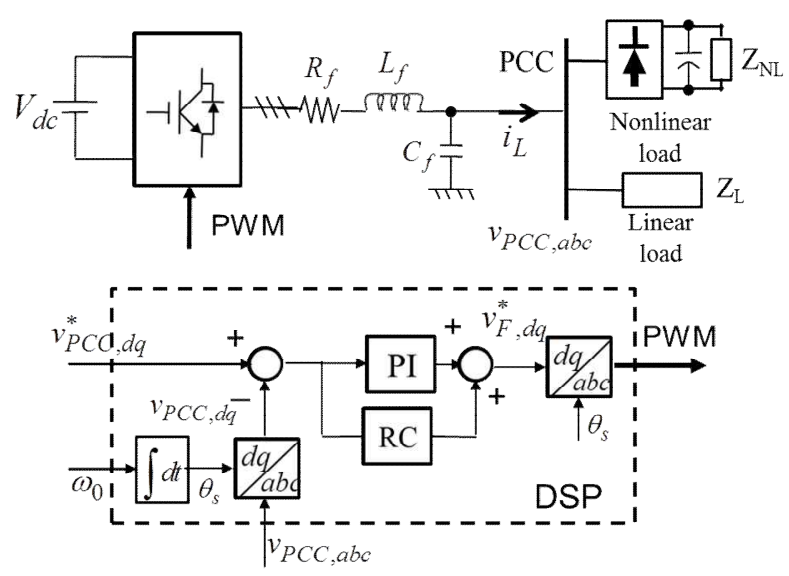

Fig. 3. Block diagram of the proposed voltage control scheme.

$$
v_{i}=v_{i 1}+\sum_{h=6 n \pm 1} v_{i h},
$$

where $v_{i h}$ is the harmonic component of the inverter output voltage.

To accurately regulate the harmonic voltage in (3), to produce a sinusoidal voltage at the PCC, the proposed voltage controller which consists of a PI and a repetitive controller is developed as shown in Fig. 3.

\section{B. Voltage Control Scheme}

The block diagram of the proposed voltage control scheme is shown in Fig. 3. This scheme consists of a PI and a repetitive controller and it is implemented in the synchronous reference $(d-q)$ frame rotating at the fundamental frequency $\omega_{0}$. In the proposed control scheme, the PI controller aims to regulate the fundamental component of the PCC voltage, whereas the purpose of the RC is to compensate the harmonic voltage drop in the line impedance in order to ensure that the PCC voltage remains sinusoidal. Therefore, the RC plays a vital role in the proposed control scheme. Without the RC, a sinusoidal voltage at the PCC cannot be achieved. However, the traditional PI-RC has a severe shortcoming. It has a very slow dynamic response due to a long time delay, which makes it difficult to maintain the PCC voltage as sinusoidal if the load conditions are varied widely and frequently. Therefore, in this paper, a new RC is proposed which has a time delay of one-sixth of the fundamental period to compensate the $(6 n \pm 1)$ th $(n=1,2,3 \ldots)$ harmonic voltage drops caused by nonlinear loads. Since the time delay of the proposed $\mathrm{RC}$ is reduced by six times compared to the traditional PI-RC, the proposed RC can provide a much faster dynamic response under load variations. The design of the $\mathrm{RC}$, which plays a crucial role in achieving good PCC voltage performance, will be presented in detail in following sections.

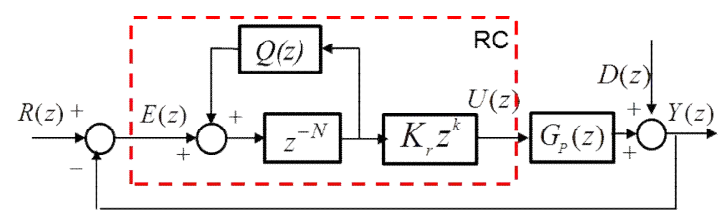

Fig. 4. Block diagram of the repetitive controller.

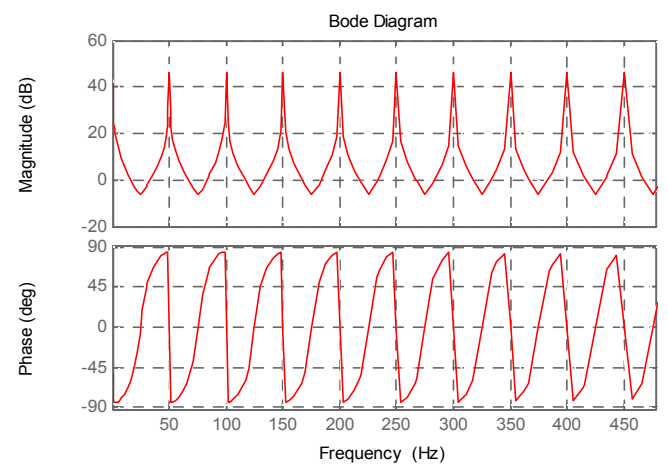

Fig. 5. Bode diagram of the traditional PI-RC.

\section{PROPOSED REPETITIVE CONTROLlER}

\section{A. Structure of Repetitive Controller}

Fig. 4 shows the general structure of a RC in the discretetime domain. Based on Fig. 4, the transfer function of the RC can be described as

$$
C(z)=\frac{U(z)}{E(z)}=\frac{K_{r} z^{-N+k}}{1-Q(z) z^{-N}},
$$

where $z^{-N}$ is the time delay unit, $z^{k}$ is the phase lead term, $Q(z)$ is a filter transfer function, $K_{r}$ is the $\mathrm{RC}$ gain, $R(z)$ is the reference value, $E(z)$ is the tracking error, and $U(z)$ is the output of the RC.

In (4), $N$ is the number of samples in one fundamental period which is defined as the ratio of the sampling frequency and the fundamental output frequency. As a result, the RC has three main components that need to be determined, i.e., the filter $Q(z)$, the phase lead term $z^{k}$, and the RC controller gain $K_{r}$

Fig. 5 shows the Bode diagram of the traditional PI-RC given in (4). In this case, the fundamental frequency is selected to be $50 \mathrm{~Hz}$. The traditional PI-RC is implemented with a time delay of one fundamental period $\left(T_{f}\right)$ which provides a high controller gain at every (odd and even) harmonic frequency, as shown in Fig. 5. Thanks to the effectiveness of the traditional PI-RC in harmonic compensation, it has been used in many applications [10]-[18].

\section{B. Proposed Repetitive Controller}

Even though the traditional PI-RC has been widely used, it still has several drawbacks: first, even though most $\mathrm{AC}$ systems have only odd harmonic components, the traditional PI-RC attempts to compensate every harmonic component, 
including even harmonics, which is waste of control effort; second, since the control action of the traditional PI-RC is delayed by one fundamental period, the dynamic response is typically too slow and is unacceptable for many applications.

In order to overcome these drawbacks, this paper proposes a new RC that can improve both the dynamic response and the steady-state performance of the RCs in three-phase systems. It is well-known that three-phase nonlinear loads produce only $(6 n \pm 1)$ th $(n=1,2,3 \ldots)$ harmonic components and the $(6 n \pm 1)$ th harmonics become $6 n$th harmonics in the $d-q$ frame. Therefore, to compensate the harmonics in three-phase systems, it is sufficient to take into account only the $6 n$th $(n=1,2,3 \ldots)$ harmonics instead of every (odd and even) harmonic as considered in (4). As a result, the transfer function of the proposed RC, which considers only $6 n$th $(n=1$, $2,3 \ldots$ ) harmonics in the $d-q$ frame, can be described as follows:

$$
C(z)=\frac{U(z)}{E(z)}=\frac{K_{r} z^{-N / 6+k}}{1-Q(z) z^{-N / 6}} .
$$

From (5), the number of sampling delays is reduced by six times compared to the traditional PI-RC given in (4). Therefore, the dynamic response of the proposed $\mathrm{RC}$ can be improved to approximately six times faster than that of the traditional PI-RC if the same values of $K_{r}, Q(z)$, and $k$ are used in both (4) and (5). In addition, by considering only the $6 n$th $(n=1,2,3 \ldots)$ in the $d-q$ frame or the $(6 n \pm 1)$ th harmonics, the proposed RC can avoid the possibility of amplifying or re-injecting other harmonics. Thus, it can enhance the steady-state performance of the PCC voltage.

In (5), the role of $Q(z)$ is to improve the system stability margin by reducing the peak gains of the $\mathrm{RC}$ in high-frequency ranges. In the traditional PI-RC, $Q(z)$ is usually selected as a close-to-unity constant for ease of implementation [10]. However, as shown in Fig. 6(a), the RC has the same peak at all of the $6 n$-th harmonic frequencies when $Q(z)$ is a close-to-unity constant. This means that the system can be unstable if these peaks are too high in the high-frequency range. Hence, in order to avoid system instability, it is usually better to reduce these peak gains, even though reduced peak gain results in a deterioration of the steady-state performance [10], [16].

In this paper, to improve the stability margin without degrading the steady-state performance of the RC, a zero phase-shift low-pass filter (LPF) with the transfer function given in (6) is used to implement $Q(z)$.

$$
Q(z)=\alpha_{1} z+\alpha_{0}+\alpha_{1} z^{-1},
$$

where $\alpha_{0}+2 \alpha_{1}=1$ and $\alpha_{0}, \alpha_{1}>0$.

As shown in Fig. 6(b), when $Q(z)$ is a LPF, the RC still produces a high gain in the low-frequency range, whereas the magnitude of the peaks in the high-frequency regions are reduced in order to ensure system stability. Even though the

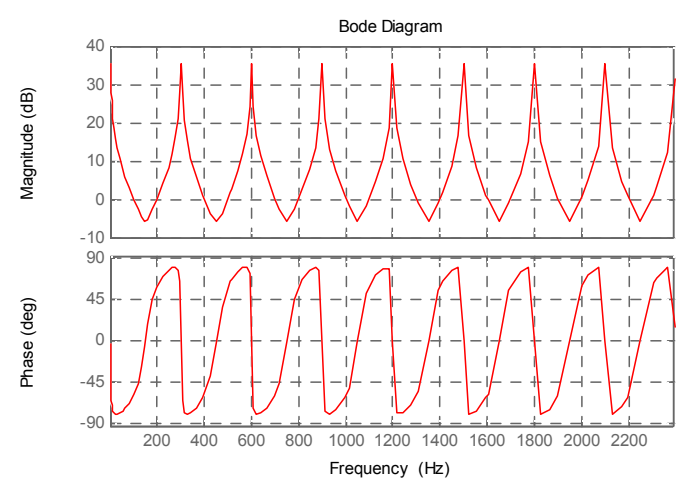

(a)

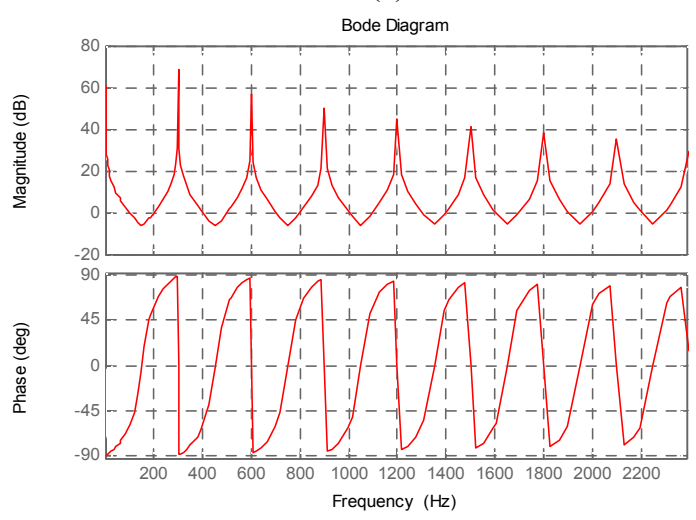

(b)

Fig. 6. Bode diagram of the $\mathrm{RC}$ where (a) $\mathrm{Q}(\mathrm{z})=0.95$ (b) $\mathrm{Q}(\mathrm{z})$ is a LPF.

LPF also reduces the peak gains in the high-frequency range, the peak gains in the low-frequency range are still high enough to ensure good harmonic compensation performance. Moreover, compensating the high-order harmonics is not important because the magnitude of the high-frequency harmonics is normally very small and negligible. Therefore, a zero-phase-shift LPF is a more suitable choice for $Q(z)$ than a close-to-unity constant since it can enhance the system stability without having an impact on the steady-state performance of the RC.

In order to investigate the stability conditions of the proposed $\mathrm{RC}$, the relationship between the tracking error $E(z)$, the reference $R(z)$, and the disturbance $D(z)$ is derived as (7) according to Fig. 4.

$$
\begin{gathered}
E(z)=\frac{\left[1-Q(z) z^{-N / 6}\right]\left[1-G_{P}(z)\right]}{1-z^{-N / 6}\left[Q(z)-K_{r} z^{k} G_{P}(z)\right]} R(z) \\
-\frac{1-Q(z) z^{-N / 6}}{1-z^{-N / 6}\left[Q(z)-K_{r} z^{k} G_{P}(z)\right]} D(z)
\end{gathered} .
$$

Let $H(z)=Q(z)-K_{r} z^{k} G_{P}(z)$. The repetitive control system is stable when the condition given in (8) is satisfied [10].

$$
\left|H\left(e^{j \omega T}\right)\right|<1
$$




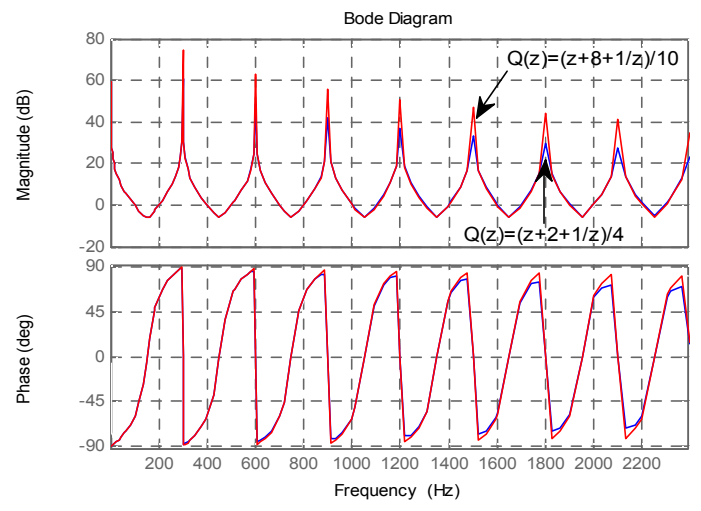

Fig. 7. Bode diagram of the repetitive controller with different LPFs.

where $\omega \in[0, \pi / T], T$ denotes the sampling period and $\pi / T$ is the Nyquist frequency.

According to (8), when $\omega$ increases from zero to the Nyquist frequency, as long as $H\left(e^{j \omega T}\right)$ is located within the unity circle, the repetitive control system is sufficiently stable. This condition is taken into account when designing the RC.

\section{Design OF THE REPETITIVE CONTROLleR}

The RC is designed for a DG system with the system parameters given in Table I. As mentioned earlier, the RC has three main components that need to be determined, i.e., the filter $Q(z)$, the phase lead term $z^{k}$, and the RC controller gain $K_{r}$.

Regarding the filter $Q(z)$, as previously discussed, a zero phase-shift LPF with the transfer function given in (6) is suitable to implement $Q(z)$. In (6), $\alpha_{0}$ should be carefully selected to achieve good performance of the RC. Fig. 7 shows the Bode diagram of the RC with two different LPFs. As shown in Fig. 7, a LPF with a larger value of $\alpha_{0}$ causes a larger cut-off frequency and a wider bandwidth, which results in better steady-state performance of the RC because a larger amount of harmonic components can pass through the LPF [13]. As a result, the LPF $Q(z)$ with the transfer function (9) is selected.

$$
Q(z)=\left(z+8+z^{-1}\right) / 10 .
$$

The phase lead term $z^{k}$ is used to compensate both the phase lag introduced by $G_{P}(z)$ and the delay caused by the calculation time of the digital control, and $k$ is selected to minimize the phase displacement of $G_{P}(z) z^{k}$. If the output of the DG is considered an ideal voltage source, the plant can be simplified as the $L C$ filter with the transfer function as

$$
G_{P}(s)=\frac{1}{L_{f} C_{f} s^{2}+R_{f} C_{f} s+1} .
$$

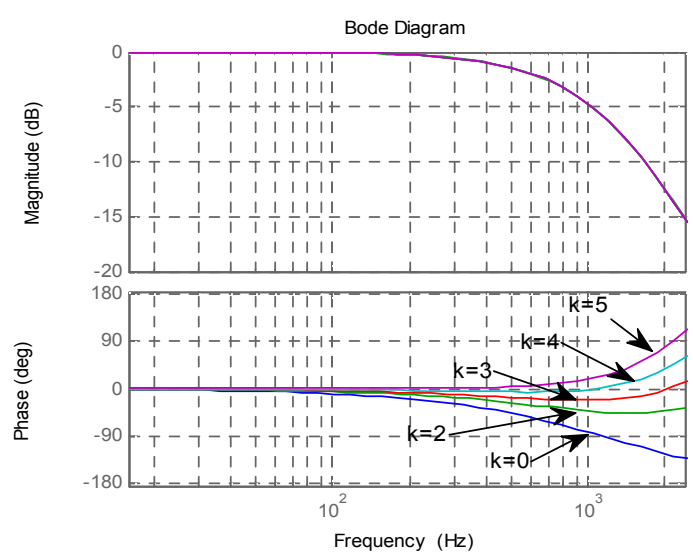

Fig. 8. Phase lag compensation with different values of $k$.

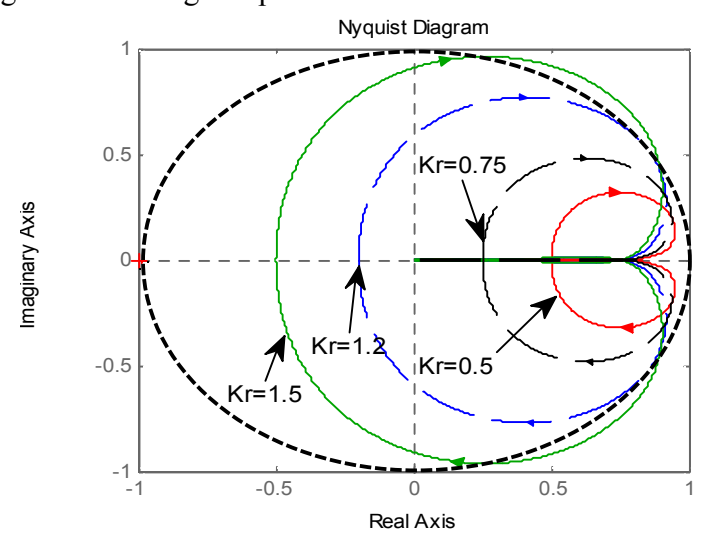

Fig. 9. Loci of vector $H\left(e^{j \omega T}\right)$ with different values of $K_{r}$.

Fig. 8 shows the Bode diagram of $G_{P}(z) z^{k}$ with different values of $k$. In Fig. 8, a minimum phase displacement is achieved with $k=4$. In addition, if one extra delay caused by the computation time of the digital control is considered, $k=5$ is selected.

After properly designing $Q(z)$ and $z^{k}$, the RC controller gain $K_{r}$ should also be carefully selected so that the stability condition given in (8) is satisfied. Fig. 9 shows the loci of the vector $H\left(e^{j \omega T}\right)$ with different values of $K_{r}$. It can be observed in Fig. 9 that the vector $H\left(e^{j \omega T}\right)$ is located inside the unity circle if $K_{r}$ is less than 1.2. In fact, a large $K_{r}$ results in a smaller steady-state error, but limits the stability margin of the system. Therefore, in order to guarantee a sufficient stability margin, $K_{r}=0.75$ is chosen.

\section{Simulation Results}

A simulation model of a DG system is built with PSIM to verify the effectiveness of the proposed controller. The DG system consists of a three-phase voltage-source-inverter, an output $L C$ filter, a linear load, and a nonlinear load as shown in Fig. 3. A DC source is used for the sake of simplicity because this study focuses on the output voltage quality of the DG. The detailed system parameters are given in Table I. 


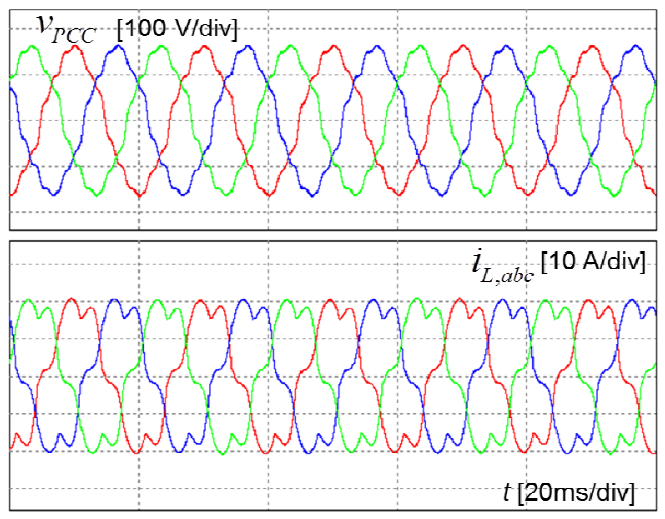

(a)
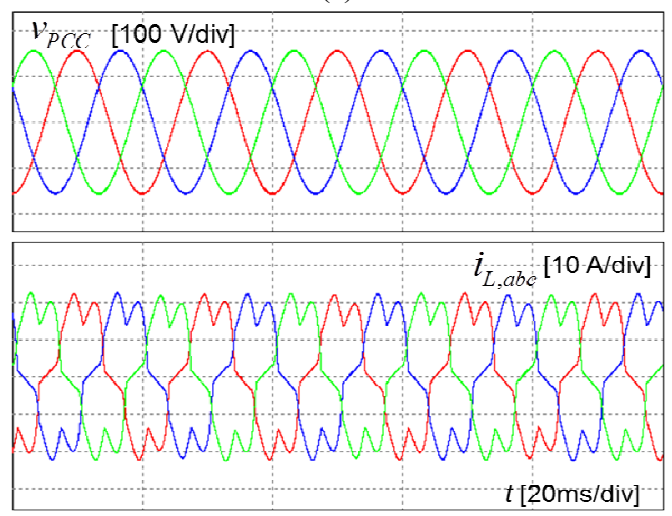

(b)

Fig. 10. Steady-state performance of the PCC voltage and current with (a) PI controller, (b) Proposed PI-RC

TABLE I

SYSTEM PARAMETERS

\begin{tabular}{|c|c|}
\hline Parameters & Values \\
\hline Reference output voltage & $110 \mathrm{~V}(\mathrm{RMS})$ \\
\hline Output frequency & $50 \mathrm{~Hz}$ \\
\hline DC-link voltage & $350 \mathrm{~V}$ \\
\hline Sampling/Switching frequency & $9 \mathrm{kHz}$ \\
\hline Rated output power & $5 \mathrm{~kW}$ \\
\hline $\begin{array}{l}\text { Number of sampling delay for the } \\
\text { proposed RC }\end{array}$ & 30 \\
\hline $\begin{array}{l}\text { Number of sampling delay for the } \\
\text { traditional PI-RC }\end{array}$ & 180 \\
\hline$L C$ filter inductance & $4 \mathrm{mH}$ \\
\hline$L C$ filter capacitance & $27 \mu \mathrm{F}$ \\
\hline Damping resistor of $L C$ filter & $0.5 \Omega$ \\
\hline Three-phase linear load $Z_{L}$ & $80 \Omega$ \\
\hline Load of diode rectifier $Z_{N L}$ & $\begin{array}{c}\mathrm{R}=30 \Omega \\
\mathrm{C}=2200 \mu \mathrm{F}\end{array}$ \\
\hline
\end{tabular}

Fig. 10 shows the steady state performance of the PCC voltage and current with the PI and the proposed controller. In Fig. 10(a), it is clear that the PI controller is unable to maintain a sinusoidal output voltage at the PCC without an additional harmonic compensator. The harmonic currents due to the nonlinear load lead to a harmonic voltage drop caused

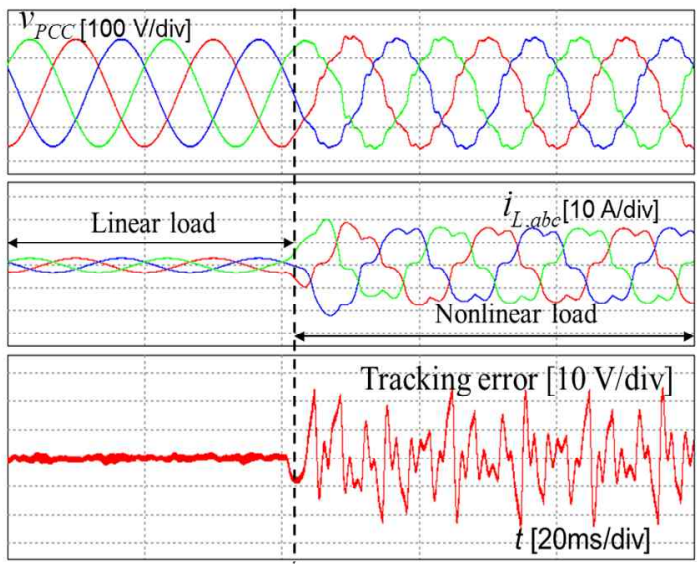

(a)

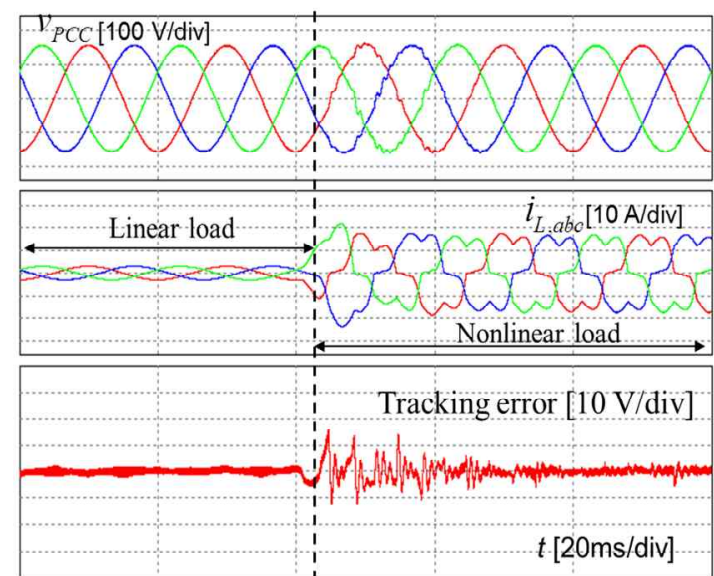

(b)

Fig. 11. Dynamic response of the PCC voltage and current when a nonlinear load is connected with (a) PI controller (b) proposed PI-RC.

by the line impedance and result in voltage distortions at the PCC. The THD value of the PCC voltage without harmonic compensation is quite high at about $7.8 \%$. In contrast, in Fig. 10 (b), thanks to the effectiveness of the proposed control scheme, the DG can supply a sinusoidal output voltage at the PCC regardless of the nonlinear load. The PCC voltage is regulated to be sinusoidal with an ultra-low THD of about $0.82 \%$. The proposed control scheme provides very good harmonic compensation performance.

Along with good steady-state performance, the fast dynamic response under load variations is also a crucial factor of the DG. Fig. 11 shows the dynamic response of the PCC voltage when a nonlinear load is connected with the PI controller and the proposed PI-RC. In Fig. 11(a), the PI controller cannot maintain a sinusoidal PCC voltage when a nonlinear load is applied. In contrast, in Fig. 11(b), the voltage at the PCC is maintained to be sinusoidal thanks to the proposed PI-RC controller. As a result, it can be said that the proposed controller is superior compared with the PI controller. 


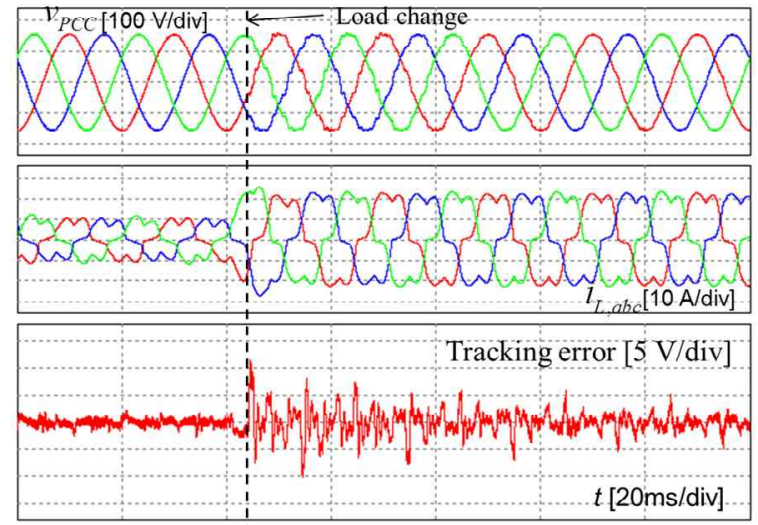

(a)

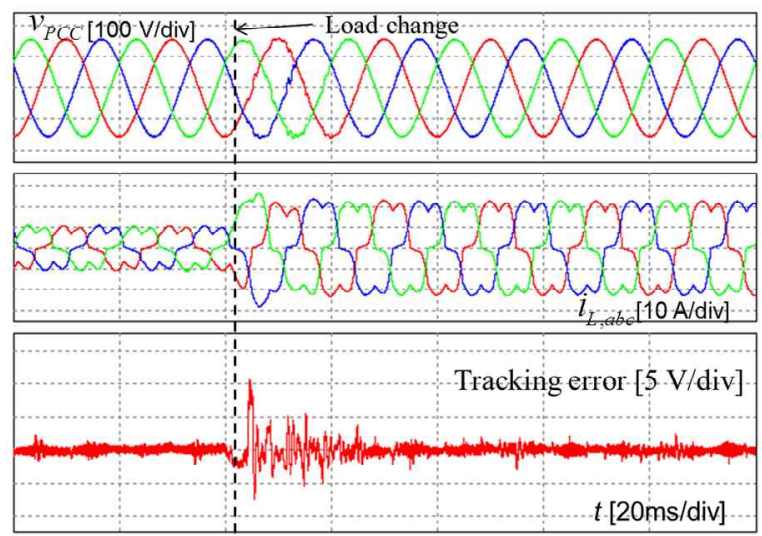

(b)

Fig. 12. Dynamic response of the PCC voltage and current with (a) traditional PI-RC, (b) proposed PI-RC.

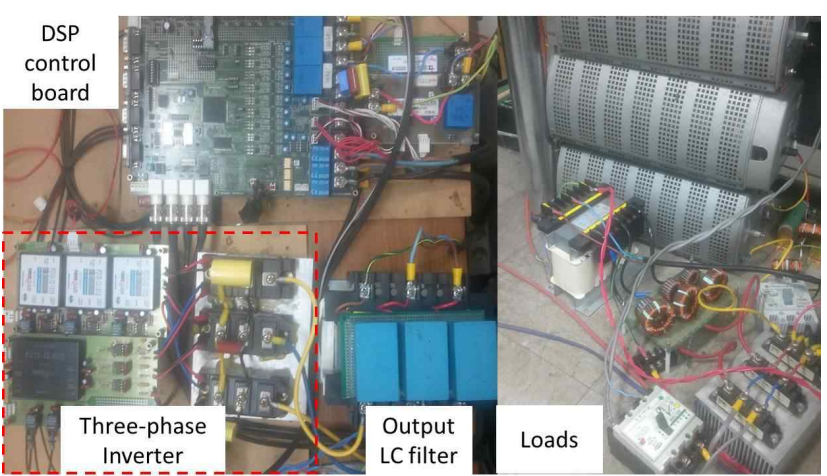

Fig. 13. Experimental setup for the DG.

The dynamic response of the DG with the traditional PI-RC [10] and the proposed PI-RC under load variations is shown in Fig. 12 when the load is changed. In Fig. 12(a), the DG needs more than $100 \mathrm{~ms}$ (five fundamental cycles) to regain a sinusoidal PCC voltage with the traditional PI-RC. Since the traditional PI-RC has a delay time of one fundamental cycle, it usually needs several fundamental cycles to reach the steady-state condition after load changes. Meanwhile, the proposed PI-RC shows a much faster dynamic response in Fig. 12(b) when compared to the traditional PI-RC. The tracking error becomes almost zero and the PCC voltage can be restored to a sinusoidal waveform within a short duration of about $20 \mathrm{~ms}$ (one fundamental cycle). From the simulated results, it can be said that the proposed controller provides excellent steady-state performance as well as a very fast dynamic response.

\section{EXPERIMENTAL RESULTS}

An overview of the experimental system is shown in Fig. 13. All of the parameters in the experimental system are the same as the ones for the simulation model given in Table I. The control strategy is carried out through a floating-point DSP (TMS320F28335 by Texas Instruments). The control and switching frequency are also set at $9 \mathrm{kHz}$.

\section{A. Steady-state Performance of the PCC Voltage under Nonlinear Load Conditions}

Fig. 14(a) shows the performance of the PCC voltage with the PI controller when a nonlinear load is connected to the system. As shown in Fig. 14(a), due to the presence of the nonlinear load, the PCC voltage cannot be sinusoidal. The total harmonic distortion (THD) of the PCC voltage under this condition is $8.1 \%$. It is obvious that the PI controller is not sufficient for improving the PCC voltage performance when nonlinear loads are used in the system.

In contrast, by using the proposed PI-RC, the PCC voltage is regulated to be sinusoidal regardless of the existence of the nonlinear load, as shown in Fig. 14(b). The THD value of the PCC voltage is very small at about $0.88 \%$. The experimental results coincide with those of simulation shown in Fig. 10.

\section{B. Dynamic Responses of the PCC Voltage when the Nonlinear Load Connects to the System}

The dynamic responses of the PCC voltage when the nonlinear load is connected to the system are shown in Fig. 15. Fig. 15(a) and (b) show the responses with the PI controller and the proposed PI-Repetitive controller, respectively. It can be seen in Fig. 15(a) that, with the PI controller, the PCC voltage is sinusoidal when the load is linear. However, when a nonlinear load is connected to the system, the performance of the PCC voltage deteriorates and the PCC voltage is no longer sinusoidal. These results reveal that the conventional PI controller is effective only when the load is linear. In other cases, the PI controller fails to maintain a sinusoidal PCC voltage.

In contrast, as shown in Fig. 15(b), with the proposed controller, the PCC voltage is regulated to be sinusoidal regardless of whether the load is linear or nonlinear. When the nonlinear load is connected, the controller needs a very short settling time of about $25 \mathrm{~ms}$ in order to recover a sinusoidal PCC voltage. The PCC voltage performance is almost unchanged even though the nonlinear load is connected. 


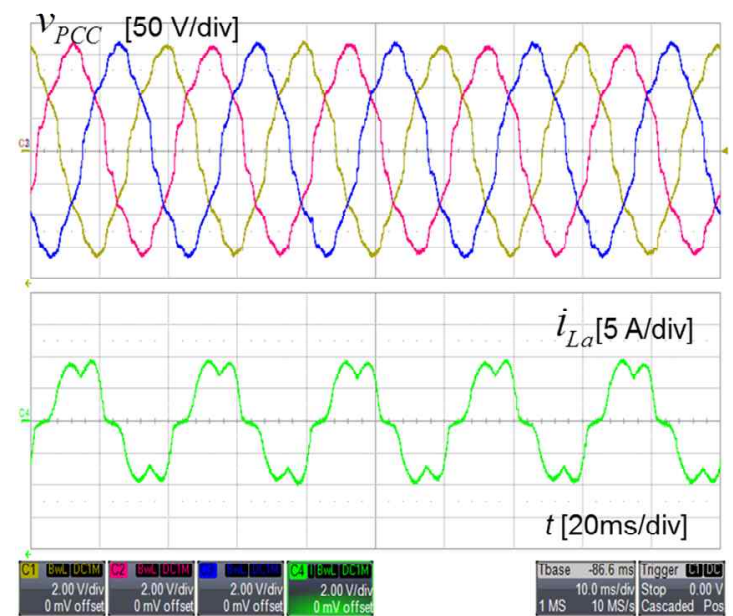

(a)
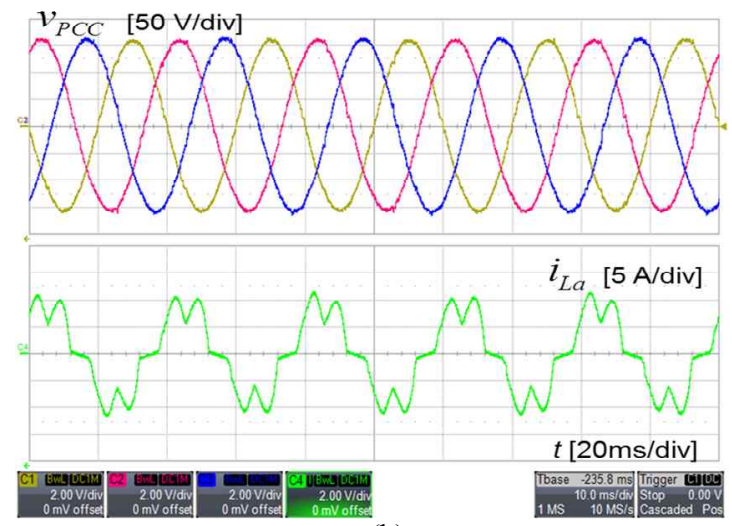

(b)

Fig. 14 Steady-state performance of the PCC voltage and current with (a) PI controller (b) Proposed PI-RC.

\section{Dynamic Responses of the PCC Voltage during Load} Changes

In order to compare the dynamic performance of the traditional PI-RC [10] with that of the proposed PI-RC, the dynamic responses of the PCC voltage when the load power is changed are illustrated in Fig. 16. Fig. 16(a) and (b) show the responses with the traditional PI-RC and the proposed RC, respectively. From Fig. 16(a), it can be observed that the traditional PI-RC has a slow dynamic response under load changes. When the load is increased, the controller needs about $80 \mathrm{~ms}$ to regain a sinusoidal PCC voltage.

Meanwhile, the proposed PI-RC provides a much faster dynamic response. As shown in Fig. 16(b), it takes only about $20 \mathrm{~ms}$ (one fundamental cycle) to recover the PCC voltage to be sinusoidal when the load is changed. The experimental results coincide with those of the simulation shown in Fig. 12. The proposed PI-RC provides a much faster dynamic response than the traditional PI-RC.

The performance of the PCC voltage is summarized in Table II where the PI-RC in [10] is adopted as a representative of the traditional PI-RC. In Table II, because the DG with the PI controller has no harmonic compensator,

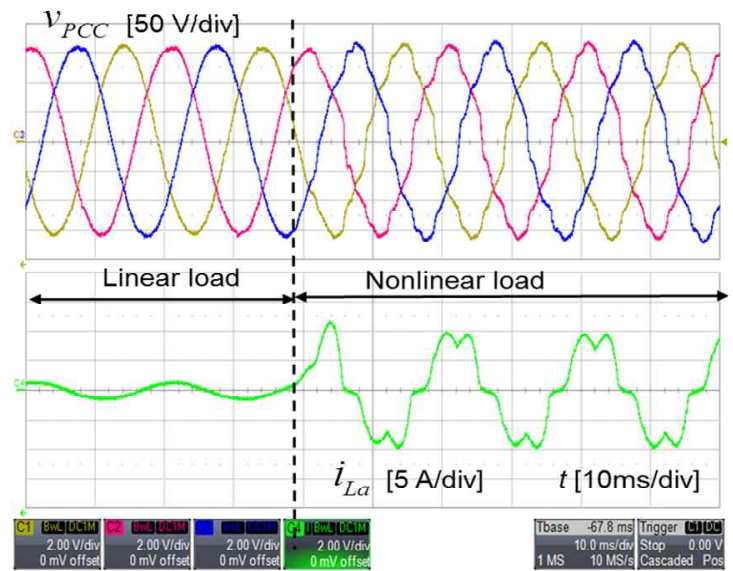

(a)

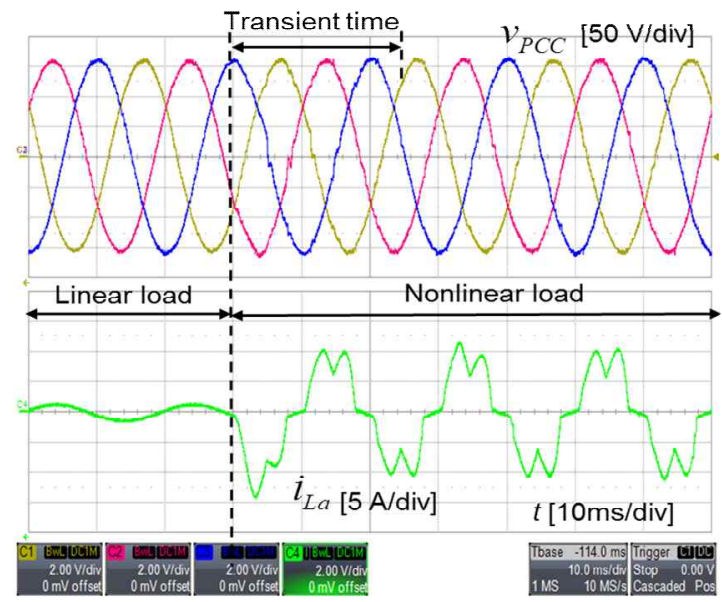

(b)

Fig. 15 Dynamic response of the PCC voltage and current when a nonlinear load is connected with (a) PI controller (b) proposed PI-RC.

it shows a very high THD value. Meanwhile, the DG with the traditional PI-RC in [10] or the proposed PI-RC has an ultra-low THD when compared to that observed with the PI controller. However, when compared to the traditional PI-RC, the proposed PI-RC achieves a slightly smaller THD as well as a much faster dynamic response. As a result, the proposed controller provides an excellent steady-state performance and a very fast dynamic response.

TABLE II

Performance Comparisons of PI CONTROLler, TRADITIONAL PI-RC, AND PROPOSED PI-RC

\begin{tabular}{|c|c|c|c|c|}
\hline & & $\begin{array}{c}\text { PI } \\
\text { controller }\end{array}$ & $\begin{array}{c}\text { Traditional } \\
\text { PI-RC [10] }\end{array}$ & $\begin{array}{c}\text { Proposed } \\
\text { PI-RC }\end{array}$ \\
\hline $\begin{array}{c}\text { THD } \\
\text { of } \\
v_{P C C}\end{array}$ & Simulation & $7.8 \%$ & $1.21 \%$ & $0.82 \%$ \\
\cline { 2 - 5 } & Experiment & $8.1 \%$ & $1.34 \%$ & $0.88 \%$ \\
\hline $\begin{array}{c}\text { Dynamic } \\
\text { response }\end{array}$ & & & $\sim 120 \mathrm{~ms}$ & $\sim 20 \mathrm{~ms}$ \\
\hline
\end{tabular}




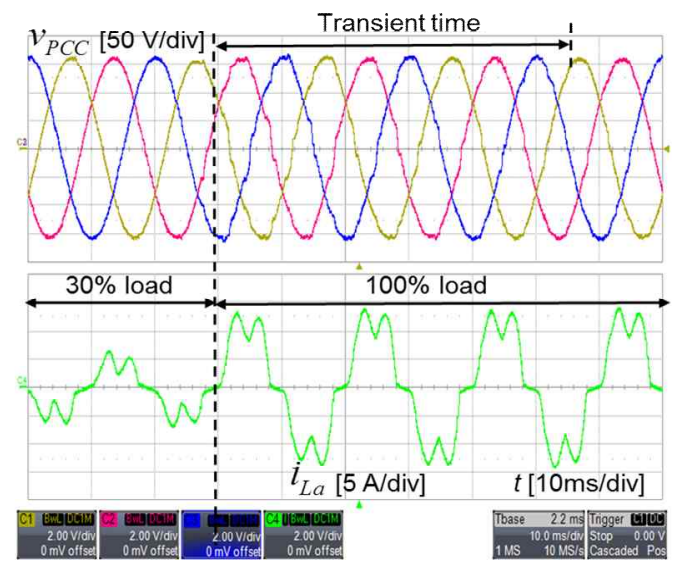

(a)

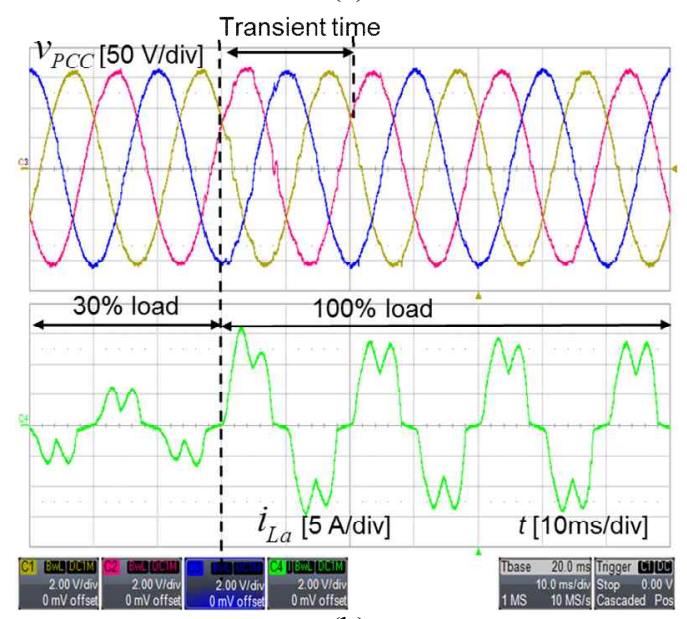

(b)

Fig. 16. Dynamic response of the PCC voltage and current when load is changed with (a) traditional PI-RC (b) proposed PI-RC

\section{CONCLUSIONS}

This paper proposed an enhanced control scheme for a stand-alone DG system which uses an advanced repetitive controller. The analysis and design of the proposed $\mathrm{RC}$ were presented in detail. By designing the $\mathrm{RC}$ to be suitable for three-phase systems, the sampling delay is reduced six times compared to the traditional PI-RC, and the dynamic response of the proposed RC is significantly improved. In addition, by utilizing a zero-phase-shift LPF for $Q(z)$ in the proposed RC, the steady-state performance of the PCC voltage is improved without any adverse effects on system stability. The proposed controller provides a very fast dynamic response and excellent steady-state performance. The effectiveness of the proposed control strategy is verified through experiments. The PCC voltage is regulated to be almost purely sinusoidal with an ultra-low THD of about $0.88 \%$ irrespective of the presence of nonlinear loads.

\section{ACKNOWLEDGMENT}

This work was partly supported by the NRF of Korea Grant funded by the Korea Government (No. 2010-0025483) and the Network-based Automation Research Center (NARC) funded by the Ministry of Trade, Industry \& Energy.

\section{REFERENCES}

[1] M. Borbely and J. F. Kreider, Distributed Generation The Power Paradigm for the New Millennium. CRC Press, 2001.

[2] F. Blaabjerg, R. Teodorescu, M. Liserre, and A.V. Timbus, "Overview of control and grid synchronization for distributed power generation systems," IEEE Trans. Ind. Electron., Vol. 53, No. 5, pp1398-1409, Oct. 2006.

[3] R. Noroozian, G. Gharehpetian, M. Abedi, and M. Mahmoodi, "Grid-Tied and Stand-Alone Operation of Distributed Generation Modules Aggregated by Cascaded Boost Converters," Journal of Power Electronics, Vol. 10, No. 1, pp.97-105, Jan. 2010.

[4] R. Bojoi, L. R. Limongi, D. Roiu, and A. Tenconi, "Enhanced power quality control strategy for single-phase inverters in distributed generation systems," IEEE Trans. Power Electron., Vol. 26, No. 3, pp.798-806, Mar. 2011.

[5] S.-H. Ko, S.-W. Lee, S.-R. Lee, C. V. Nayar, and C.-Y. Won, "Design considerations for a distributed generation system using a voltage-controlled voltage source inverter," Journal of Power Electronics, Vol. 9, No. 4, pp.643-653, Jul. 2009.

[6] R. Teodorescu and F. Blaabjerg, "Flexible control of small wind turbines with grid failure detection operating in stand-alone or grid-connected mode,"IEEE Trans. Power Electron., Vol. 19, No. 5, pp. 1323-1332, Sep. 2004.

[7] Z. Chen, Y Hu, and F. Blaabjerg, "Control of distributed power systems" in Proceedings of International Power Electronics and Motion Control Conference 2006 (IPEMC '06), pp. 1-6, Aug. 2006.

[8] H. Patel and V. Agarwal, "Control of a stand-alone inverter-based distributed generation source for voltage regulation and harmonic compensation," IEEE Trans. Power Del., Vol. 23, No .2, pp. 1113-1120, Apr. 2008.

[9] H. Nian and R. Zeng, "Improved control strategy for stand alone distributed generation system under unbalanced and non linear loads," IET Renew. Power Gener, Vol. 5, No. 5, pp. 323-331, Sep. 2011.

[10] K. Zhang, Y. Kang, J. Xiong, and J. Chen, "Direct Repetitive Control of SPWM Inverters for UPS Purpose," IEEE Trans. Power Electron., Vol. 18, No. 3, pp. 784-792, May 2003

[11] P. C. Loh, Y. Tang, F. Blaabjerg, and P. Wang, "Mixed-frame and stationary-frame repetitive control schemes for compensating typical load and grid harmonics," IET. Power Electron., Vol. 4, No. 2, pp. 218-226, Feb. 2011.

[12] B. Zhang, D. Wang, K. Zhou, and Y. Wang, "Linear phase lead compensation repetitive control of a CVCF PWM inverter," IEEE Trans. Ind. Electron., Vol. 55, No. 5, pp. 1595-1602, Apr. 2008.

[13] K. Zhou, K.-S. Low, D. Wang, F.-L. Luo, B. Zhang, and Y. Wang, "Zero-phase odd-harmonic repetitive controller for a single-phase PWM inverter," IEEE Trans. Power Electron., Vol. 21, No. 1, pp. 193-201, Jan. 2006. 
[14] G. Escobar, J. Leyva-Ramos, P. R. Martınez, and A. A. Valdez, "A repetitive-based controller for the boost converter to compensate the harmonic distortion of the output voltage," IEEE Trans. Control Syst. Technol., Vol. 13, No. 3, pp. 500-508, May 2005.

[15] Cho. Y and Lai. J, "Digital plug-in repetitive controller for single-phase bridgeless PFC converters," IEEE Trans. Power Electron., Vol. 28, No. 1, pp. 165-175, Jan. 2013.

[16] Y. He, J. Liu, J. Tang, Z. Wang, and Y. Zou, "Deadbeat control with a repetitive predictor for three-level active power filters," Journal of Power Electronics, Vol. 11, No. 4, pp.583-590 , Jul. 2011.

[17] A. Garcia-Cerrada, O. Pinzon-Ardila, V. Feliu-Batlle, P. Ron-cero-Sanchez, and P. Garcia-Gonzalez, "Application of a repetitive controller for a three-phase active power filter," IEEE Trans. Power Electron., Vol. 22, No. 1, pp. 237-246, Jan. 2007.

[18] R. Grino, R. Cardoner, R. Costa-Castello, and E. Fossas, "Digital repetitive control of a three-phase four-wire shunt active filter," IEEE Trans. Ind. Electron., Vol. 54, No. 3, pp. 1495-1503, Jun. 2007.

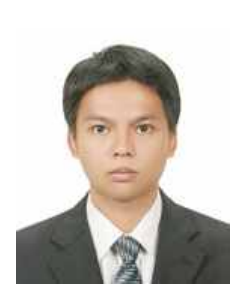

Quoc-Nam Trinh was born in Thanh-Hoa, Vietnam, in 1985. He received his B.S. in Electrical Engineering from the Ho Chi Minh City University of Technology, Ho Chi Minh City, Vietnam, in 2008. Currently, he is an M.S./Ph.D. combined student at the University of Ulsan, Ulsan, Korea. He is a member of the Korean Institute of Power Electronics (KIPE). His current research interests include distributed generations, wind power systems, active power filters, and power quality.

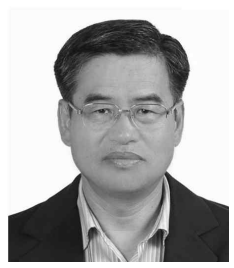

Hong-Hee Lee received his B.S., M.S., and $\mathrm{Ph} . \mathrm{D}$. in Electrical Engineering from Seoul National University, Seoul, Korea, in 1980, 1982, and 1990, respectively. $\mathrm{He}$ is a Professor in the School of Electrical Engineering, University of Ulsan, Ulsan, Korea. $\mathrm{He}$ is also the Director of the Network-based Research Center (NARC), University of Ulsan. His current research interests include power electronics, network-based motor control, and control networks. He is a member of the Institute of Electrical and Electronics Engineers (IEEE), the Korean Institute of Power Electronics (KIPE), the Korean Institute of Electrical Engineers (KIEE), and the Institute of Control, Automation, and Systems Engineers (ICASE). 